\title{
Editorial
}

\section{Policy and procedures on scientific misconduct}

\author{
G Melino ${ }^{*, 1}$ on behalf of the Editorial Board \\ Cell Death and Differentiation (2010) 17, 1805-1806; doi:10.1038/cdd.2010.122
}

One negative side effect of scientific competition is the temptation to knowingly manipulate images and statistics and even to deliberately falsify data. We believe, along with the vast majority of scientists, that this behaviour is scientifically pathological and totally contrary to the spirit of honest rigorous experimentation and its equally frank reporting. We also think that bench scientists, authors, referees, editors, readers, and publishers have an obligation to uphold the highest standards of scientific integrity.

Publishers and editors of scientific journals play particularly important roles in maintaining these standards. ${ }^{1}$ Errors will inevitably occur for several reasons: inadvertently, due to the statistical nature of data; accidentally, due to carelessness or incompetence; or deliberately, due to fabrication or falsification of results. To enhance the integrity of the literature, and to increase the speed with which science progresses, it is important that, by ensuring good practice, ${ }^{2,3}$ errors are identified and corrected efficiently, whether their cause is innocent or intentional. As Editors, occasionally, but with increasing frequency, we receive reports of scientific misconduct, ranging from fabricated, fraudulent or 'beautifying' image manipulation, to incoherent statistical analysis and plagiarism, not to mention 'sloppy science'. Cell Death and Differentiation $(C D D)$ is committed to publishing clean science, and we will continue to put all our efforts into assuring the validity of the data in the papers we publish. Moreover, $C D D$ is a member of the Committee On Publication Ethics (COPE) and we are obliged to follow their guidelines where there is reasonable doubt about the quality of the data. Here, we would like to briefly describe our policy on this issue.

Even though they might not necessarily cause errors to enter the literature, it is also important that other forms of misconduct, such as plagiarism or honorary authorship, are subject to sanctions so that researchers have confidence in the fairness of the scientific enterprise.

Prevention requires Editorial screening during the review process, and careful analysis by the receiving editor as well as by the referees; upon acceptance every paper is screened for plagiarisms. In order for publishers and editors to correct errors by publishing corrections or rebuttals or retracting publications, they first have to be alerted to a potential problem. Once a paper is published, it is readers of the paper, or scientists trying to replicate the results, who will first notice a potential problem. When there is a concern, it is only by taking action, such as contacting the journal editors or the authors of the paper, that it will be possible to determine whether the suspicions are justified and, if so, who is responsible, and for remedial action to be taken.

The quality and integrity of the scientific literature thus depends not only on the authors of a publication, but also on the readers of the paper and the editors and publishers of the journal.

In this issue of $C D D$, there appears a retraction ${ }^{4}$ of a paper first published in the February 2009 issue. ${ }^{5} \mathrm{~A}$ vigilant reader noticed that some of the images of blots were suspicious, with apparent discrepancies in some of the figures, including the potential addition or removal of individual lanes, and possibly re-using the same blots but comparing them with different loading controls. The reader contacted the editors of $C D D$ and explained their concerns. In this particular case, we first asked the advice of an internal commission of three senior $C D D$ editors as well as the receiving editor of the paper. Based on their response, we then requested the original data from the authors together with an explanation of how these apparent discrepancies could have arisen, which would be reassessed by the same internal commission.

The authors took action immediately. They obtained and examined all of the original data and acknowledged the problems raised. The corresponding author took responsibility and action. Because the laboratory had kept all of the original data, it was possible for them to provide us with a set of blots and a potential explanation. Although there may be a perfectly genuine explanation for the appearance of the images in the figures, it was not possible for the commission to determine this with certainty, or, if the images had indeed been manipulated illegitimately, to determine who was responsible. To get to the bottom of this matter, it has been necessary for action to be taken by someone who has the necessary legal authority and has access to the primary data (in practice, the host institution), with a request that this matter be handled according to the National University of Singapore Research Integrity Code. Although at the time of writing the investigation has not been completed, the authors have now agreed that it is in everyone's interest for the paper to be formally retracted.

Thus, in this episode, the errors have been identified, and a proper investigation has been initiated, which will eventually correct any misleading results. In such cases, any author who creates inappropriate images in their figures should be counselled and, we dare say, be given the opportunity to learn a great deal about how figures should and should not be prepared. The senior author should learn how to prevent the 
problems and should show intellectual honesty in taking rapid action, accepting responsibility, and committing to a closer supervision of his lab members in the future.

The Editors of $C D D$ would like to express our deep gratitude to the vigilant reader, who was not only sharp enough to notice the problems, but also took the initiative to contact the editors with their concerns. We are also grateful to the authors of the paper in question and the host institution, who reacted promptly and are taking corrective action.

We believe that, in many ways, this is an example of science at its best: mistakes and errors, whether they are deliberate, accidental, or completely innocent, will inevitably arise. In this case, they have been identified, action is taking place, and all parties will have learnt and benefited from the experience.

To err (and to compete) is human, but the identification and correction of error is mandatory for the health of science. We urge all our readers to contact us if they have doubts regarding data in the papers appearing in $C D D$ that may have been manipulated in an illegitimate manner or that are contrary to the Instructions to Authors. Protecting the integrity of the literature, by correcting errors, publishing rebuttals, or retracting papers, depends on the willingness of authors, readers, editors, and publishers to take action when they have concerns, and can only serve to increase public confidence in science as a legitimate philosophical enterprise.

1. Anonymous. Under suspicion. Nature 2010; 464: 1245.

2. Research Councils of UK. Policy and Code of Conduct on the Governance of Good Research Conduct (http://www.rcuk.ac.uk/review/grc/default.htm).

3. Cumming G et al. J Cell Biol 2007; 177: 7-11.

4. Retraction. Cell Death Differ 2010; 17: 1944.

5. Huang $Q$ et al. Cell Death Differ 2009; 16: 264-277. 